\title{
Changing Environmental Influences on Substance Use Across Development
}

\author{
Danielle M. Dick,' Jason L. Pagan,' Richard Viken, ${ }^{2}$ Shaun Purcell, ${ }^{3}$ Jaakko Kaprio, ${ }^{4}$ Lea Pulkkinen, ${ }^{5}$ \\ and Richard J. Rose ${ }^{2}$ \\ ' Washington University, St Louis, Missouri, United States of America \\ ${ }^{2}$ Indiana University, Bloomington, United States of America \\ ${ }^{3}$ Whitehead Institute, MIT, Massachusetts, United States of America \\ ${ }^{4}$ University of Helsinki and National Public Health Institute, Helsinki, Finland \\ ${ }^{5}$ University of Jyväskylä, Finland
}

In n contrast to many phenotypes that have been studied using twin designs, substance use shows considerable evidence of environmental influence. Accordingly, specifying the relevant environments and understanding the nature of their effects is an important research priority. Twin studies also have demonstrated that the importance of genetic and environmental influences varies across development for a variety of behavioral outcomes, including substance use. Here, we report analyses exploring moderating effects associated with parenting and peer characteristics on adolescent smoking and drinking, measured at ages 14 and 17. We find significant evidence of moderating effects associated with two dimensions of parenting (parental monitoring and time spent in activities with parents) on adolescent smoking, measured at two time points across development, but no moderating effects on adolescent drinking. Genetic influences on smoking increased, and common environmental effects decreased, as adolescents reported less parental monitoring and spending more time with their parents. Conversely, we find evidence that adolescent drinking is more strongly influenced by peer characteristics. The importance of genetic predispositions was increased among adolescents who reported more friends who used alcohol. These analyses illustrate the importance of incorporating measured aspects of the environment into genetically informative twin models to begin to understand how specific environments are related to various outcomes. Furthermore, they illustrate the importance of using a developmental perspective to understand how specific influences may vary across different ages, and across different phenotypes.

One of the most interesting findings to emerge from longitudinal studies of behavior across development is the dramatic changes that are evident in the importance of genetic and environmental influences at various stages in the lifespan. Changes in the importance of genetic and environmental influences across development have been documented across multiple behavioral domains including intellectual abilities (Bouchard \& McGue, 2003), depression (Boomsma et al., 2005), eating behavior (Klump et al., 2003), and longevity (Hjelmborg et al., 2006). This paper will focus on substance use, another area where dramatic changes in the relative importance of genetic and environmental effects across development are apparent. This has been documented in data from our longitudinal Finnish Twin Studies, where we have found that the importance of genetic effects on drinking patterns increases dramatically from adolescence to young adulthood. At age 14, genetic influences accounted for only $18 \%$ of the variance in drinking initiation and this was significant only in girls, with no evidence of genetic influence on drinking patterns in boys yet at this early age (Rose, Dick, Viken, Pulkkinen, et al., 2001). However, by age 16 genetic factors accounted for a third of the variation in drinking patterns in both sexes, and by age 18 genetic factors accounted for half of the variation (Rose, Dick, Viken, $\&$ Kaprio, 2001). Thus, over a period of just over 4 years, genetic influences changed from having virtually no detectable effect on drinking patterns to accounting for the majority of the variance. Conversely, the importance of common environmental effects decreased significantly from adolescence into adulthood, accounting for greater than $70 \%$ of the variance at age 14 , but only $\sim 15 \%$ of the variance by age 18 . Thus, as drinking patterns develop, differentiate, and stabilize across adolescence, genetic factors assume increasing importance on drinking patterns; however, alcohol use early in adolescence appears to be almost entirely influenced by family, school, and neighborhood influences (Rose et al., 2003). Interestingly, longitudinal analyses of the FinnTwin16 data suggest that not only did the overall magnitude of common environmental factors decrease

Received 6 November, 2006; accepted 29 January, 2007.

Address for correspondence: Danielle M. Dick, Washington University in St Louis, Department of Psychiatry, Box 8134, 660 South Euclid Ave, St. Louis, MO 63110, USA. E-mail: dickd@wustl.edu 
across adolescence, the relevant environmental influences on drinking frequency also changed across this age range, with environmental factors at ages 16, 17, and 18.5 being largely age-specific (Rose, Dick, Viken, $\&$ Kaprio, 2001).

However, these dramatic shifts in the importance of etiological factors are not evident across all forms of substance use. Although we found evidence of large changes in the relative importance of genetic and common environmental effects on patterns of alcohol use from age 16 to 18 (Rose, Dick, Viken, \& Kaprio, 2001), we found no changes in the importance of genetic and environmental effects on smoking patterns across this age range in the sample (Dick et al., 2006). Genetic factors consistently accounted for approximately $50 \%$ of the variance in smoking, common environment for $30 \%$, and unique environment for $20 \%$ across late adolescence. Also in contrast to our results for drinking patterns, the best fitting model for smoking patterns suggested a single common environmental factor influencing smoking across late adolescence, indicating that the environmental factors that impact smoking across this age range are more long-standing and less transient (Dick et al., 2006).

As we expand our twin models to incorporate specific measures of the environment, rather than simply modeling environmental influences latently (Dick \& Rose, 2002), the aforementioned results suggest that taking a lifespan perspective will be critical, as the influence of particular environments may vary across different points in development and show different patterns of effect depending on the behavior under study. Potential developmental changes must be identified in order to formulate a more complete understanding of the risk associated with particular environmental factors, and how this information can be used to inform prevention and intervention efforts.

With considerable evidence for an important environmental component influencing substance use in adolescence, as reviewed above, we have been focusing efforts on studying what specific environments may be important in the development of smoking and drinking patterns. Two of the most widely studied environments in relation to adolescent substance use are parental and peer influence (Barnes et al., 2000; Borawski et al., 2003; Marshal \& Chassin, 2000; Rai et al., 2003; Steinberg et al., 1994; Windle, 2000). Although the direct effects associated with these factors are often modest, we have been interested in whether the influence of parents and peers may be more apparent in interactions that modulate children's dispositional tendencies. In initial analyses, we found that parental monitoring significantly moderated genetic and environmental influences on adolescent's smoking patterns at age 14 (Dick et al., in press). Among adolescents who reported high levels of parental monitoring, genetic influences had only small effects, while environmental influences were predominant in the etiology of adolescent smoking.
Conversely, at low levels of parental monitoring, genetic influences assumed far greater importance on adolescent smoking.

Here we extend those analyses in a number of ways: We examined the influence of parental monitoring on adolescent drinking frequency at age 14 (parallel to the models previously reported for smoking), and we tested a second dimension of parenting, time spent in shared activities with parents, in relation to both smoking and drinking patterns. In addition, we examined the extent to which parental substance use may be playing a role in the effects observed with parenting practices and adolescent substance use. After fitting these models to age 14 substance use, we subsequently tested the extent to which these parenting variables were important in age 17 substance use. Finally, we report initial analyses examining the extent to which peer substance use may play a role in age 17 substance use.

\section{Methods}

\section{Participants and Procedures}

FinnTwin12 (FT12) is a population-based, developmental twin study of health-related behaviors and correlated risk factors (Kaprio et al., 2002). It consists of five consecutive birth cohorts (1983-1987) of twins identified in Finland's central population registry (CPR), permitting exhaustive and unbiased ascertainment of all twins living and resident in the country. Questionnaires were mailed to all eligible families in 1994, of which 2724 families $(87 \%)$ completed the initial family questionnaire. Immediately on receipt of the completed family questionnaire, individual questionnaires were mailed to both co-twins and both their parents (including parents not residing with either twin child). The twins' self-report questionnaires were mailed in the late autumn of the year in which the consecutive birth cohorts reached age 11 , and most twins returned their questionnaires by the first month(s) of the year they turned age 12 . Twins were sent a follow-up questionnaire in the month that they reached age 14 , and $\sim 90 \%$ of twins completed and returned it. Mean age at response was 14.05 years, with $95 \%$ responding by age 14.2 years. A second follow-up was initiated in autumn of 2000 and completed in the spring of 2005 , with questionnaire assessments of the twins at age $171 / 2$ years. The response rate among participating twins at this second follow-up was $92 \%$. The mean age at response was 17.6 years with $95 \%$ responding before they reached age 18. Assessments of nonresponders at each stage uncovered no evidence of biased selection for family structure, parental age, area of residence, or twins' zygosity or sex.

Zygosity was determined using a well-validated questionnaire completed by both co-twins at the baseline, containing items regarding similarity and confusability (Kaprio et al., 1995). Because these twins were younger than in previous Finnish studies, 
classification was supplemented by parental response to items developed for zygosity classification of twin children (Goldsmith, 1991). Assignment of same-sex co-twins, whose zygosity could not be determined definitively from information in twin and parental questionnaires, was supplemented by comparisons of school photographs and additional information obtained from twins' mothers. Definitive zygosity diagnosis of a small group $(<5 \%)$ of same-sex twins awaits genotyping, and these twins were excluded from analyses reported here. The sample used in the age 14 analyses reported here consisted of 2918 samesex twins of confirmed zygosity: 692 monozygotic (MZ) and 777 dizygotic (DZ) male twins, and 749 MZ and $700 \mathrm{DZ}$ female twins. The sample used in the age 17 analyses consisted of 2422 same-sex twins of confirmed zygosity: $528 \mathrm{MZ}$ and $635 \mathrm{DZ}$ male twins, and $667 \mathrm{MZ}$ and $592 \mathrm{DZ}$ female twins. Preliminary power analyses suggested that there was low power to discriminate sex effects, due to the large sample sizes necessary for adequate power to detect moderating effects with ordinal outcomes. Accordingly, female and male twins were collapsed by zygosity in modeling, though thresholds for variables were allowed to differ between the sexes when indicated by the data.

\section{Measures \\ Parental Monitoring.}

Monitoring was assessed with three questions included in the twins' questionnaires at the age 11 to 12 baseline and the age 14 follow-up. The questions, created by Chassin et al. (1993), asked the adolescents to report on the degree to which their parent(s) discuss with them their daily plans, know of their interests and activities, and know their whereabouts and the identity of their associates when they are not at home; responses were made on a 4-point scale from 'almost always' to 'almost never'. These were reverse coded so that higher scores indicated greater monitoring. At the age-12 baseline, the twins' report of parental monitoring ranged from 3 to 12 , with a mean score of 10.8 $(S D=1.4)$. The range at age 14 was also 3 to 12 , with an average parental monitoring score of $10.3(S D=$ 1.6). We note that although we refer to this measure as parental monitoring, it actually does not distinguish between the extent to which parents are soliciting information about the whereabouts and activities of their children and the extent to which parents have knowledge of their children's activities due to spontaneous disclosure on the part of the child (Kerr \& Stattin, 2000; Stattin \& Kerr, 2000).

\section{Time Spent in Activities With Parents}

The twin's baseline and age 14 follow-up questionnaires contained an item that asked the twins to report on how often they did the following six things with their parents: communicate/converse; go to cultural events, the theater, movies, and so forth; do sports; favorite hobbies; trips, travels, visiting; and outdoor recreation (Narusk \& Pulkkinen, 1994). Each activity was rated on a 5-point scale ranging from 'every day' to 'never'. The scale has a coefficient alpha of .73. Because some twins omitted a particular activity, data was utilized from twins who reported at least four of the six items. The mean score for all available items was multiplied by 6 to yield a sum score. In the baseline assessment, the scores ranged from 6 to 28.5 in our sample, with a mean of $19.6(S D=3.7)$; at the age 14 assessment, the scores ranged from 6 to 30, with a mean of $16.8(S D=4.2)$. Scores were approximately normally distributed across both waves of assessment.

\section{Parental Alcohol Problems and Smoking}

The baseline parental questionnaires included a 9-item diagnostic screen for alcohol-related problems, the Malmö-modified MAST (Mm-MAST; Seppa et al., 1990), to which we added two additional items to enhance its predictive association with DSM-diagnoses of alcoholism. Because some parents omitted a small number of the MAST questions, data was used from individuals who completed nine of the 11 items, in order to minimize missing data and maximally utilize the data available. The mean score for all available items was multiplied by nine to yield a sum score. All items were scored yes (1)/no (0). MAST scores ranged from 0 to 11 . The highest MAST score for either available parent was used in modeling (mean $=3.92$, $S D=2.97)$.

Because the MAST assesses lifetime alcohol problems, scores are not necessarily representative of the exposure of the adolescents to the parental behavior. Accordingly, we also analyzed current binge drinking as reported in the parents' questionnaires. Parents reported on how often they currently 'drink more than five bottles of beer or more than a bottle of wine or more than a half of bottle of strong liquor (or comparable amount of other alcoholic beverages)' on a single occasion, using a 9-point scale ranging from 'never' to 'daily'. As with the MAST, the highest score for either available parent was used in modeling (mean $=3.93$, $S D=2.21$ ).

In the baseline questionnaires, parents also reported on whether they had ever smoked regularly, defined by daily or nearly daily smoking, and whether they still smoked regularly. Twin pairs were classified as coming from a smoking family if either parent reported regular smoking. Twin pairs for which data were available from only one parent, with that parent reporting that (s)he did not smoke regularly, were considered unknown. Of the same-sex twin pairs used in analyses, $77.6 \%$ came from families in which at least one parent had been a regular smoker at some point; $42.6 \%$ of pairs had a parent that currently smoked regularly.

\section{Adolescent Alcohol Use}

Alcohol use was measured in the age 14 questionnaire with an item that asked 'How often do you drink alcohol at all?'. This question was adapted from a ninealternative response item in Finland's biennial 
Adolescent Health Habits Survey [AHHS; (Rimpela et al., 1988)], an item used also in an earlier study of 16year-old Finnish twins (Rose et al., 1999). The wording of the question remained unchanged, but the responses were truncated to four alternatives more appropriate for this younger age group. These alternatives ranged from abstinence ('Never; I don't drink alcohol') to drinking weekly or more often. Sixty-six per cent of twins reported never drinking alcohol; $20 \%$ reported drinking less than once a month; $12 \%$ reported drinking about 1 to 2 times a month; and 3\% reported drinking weekly or more often.

It is important to clarify how our Finnish twins apparently interpreted this question on their drinking at age 14 . We administered a questionnaire at age 11 to 12 , to a subset of twins that are intensively studied in FT12; the questionnaire, administered in school following a peer nomination exercise (Pulkkinen et al., 1999), contained a set of questions on alcohol use not asked of the full sample at baseline 6 months earlier. One of the drinking questions asked of this subsample of 11- to 12-year-old twins was 'Have you ever drunk alcohol?'. Only $40 \%$ of the 11 - to 12 -year-old respondents replied 'Never; I've not even tasted it'. But, to a second question, of much greater risk-relevance for later drinking by the twins, 'Have you ever drunk alcohol with your friends, without adults around?', only $7 \%$ replied positively. Because fewer twins reported using alcohol at age 14 than had reported having tasted alcohol at age 11 to 12 , the response 'I don't use alcohol' does not imply they have never tasted it, perhaps at parents' invitation on a special occasion. It must mean not using alcohol, with some frequency and, perhaps, with one's friends and without parental knowledge or permission. We interpret abstinence, self-reported at age 14, to mean that alcohol is not used, not that it has never been tasted (Rose, Dick, Viken, Pulkkinen, et al., 2001).

Alcohol use was assessed in the age 17 follow-up questionnaire with an expanded item that asked, 'How often do you use alcohol?' followed by nine response options, ranging from 'I don't use alcohol' to 'Daily'. Very few adolescents reported daily alcohol use at 17 years of age, so this highest response option was collapsed into the second highest option, ' 2 times weekly'. As a result, a total of eight response options ranging from zero (I don't use alcohol) to seven (2 times weekly or more) were retained for analyses. By age 17, only $12 \%$ of twins reported abstinence; $5 \%$ reported use once a year or less frequently; $9 \%$ reported use 3 to 4 times a year; $10 \%$ reported use about once every two months; $14 \%$ reported use about once a month; $28 \%$ reported use a couple times a month; $16 \%$ reported use about once a week; and $7 \%$ reported use two or more times a week.

\section{Adolescent Smoking}

In both the age 14 and age 17 follow-ups, adolescent smoking was assessed with a multipart question that first asked 'Have you ever smoked (or tried smoking)?' to which adolescents responded yes or no. Adolescents who responded yes subsequently answered a question that asked 'How many cigarettes have you smoked altogether up to now?'. At age 14, the four response options were as follows: 'only one', 'about 2-10', 'about 11-50', 'over 50'. At age 17, the response options were expanded to the following: 'only one', 'about 2-10', '11-50', '51-100', 'over 100'. At each age the two smoking items were collapsed to form one variable, with five alternative responses at age 14 and six alternative responses at age 17 , in which 0 represented the individuals who reported never trying smoking, 1 represented individuals who had only smoked 1 cigarette, 2 represented individuals who reported smoking 2 to 10 cigarettes, and so forth. In this way, the smoking variable more closely paralleled the drinking variable, in which $0=$ 'never using the substance', with the subsequent responses giving an indication of the amount of use among those who had initiated. At age 14, approximately $59 \%$ of the twins reported never smoking; $14 \%$ reported smoking only one cigarette; $13 \%$ reported smoking 2 to 10 cigarettes; $6 \%$ reported smoking 11 to 50 cigarettes; and $8 \%$ reported smoking over 50 cigarettes. At age 17, 29\% reported never smoking; $8 \%$ reported smoking only one; $13 \% 2$ to 10 cigarettes; $11 \% 11$ to 50 cigarettes; $5 \% 51$ to 100 cigarettes; and 35\% over 100 cigarettes.

\section{Alcohol Use Among Friends}

At age 17, the adolescents were asked 'Do any of your friends drink?' with four response options: 'none', 'one', '2-5', 'more than five'. Approximately $18 \%$ of adolescents reported that none of their

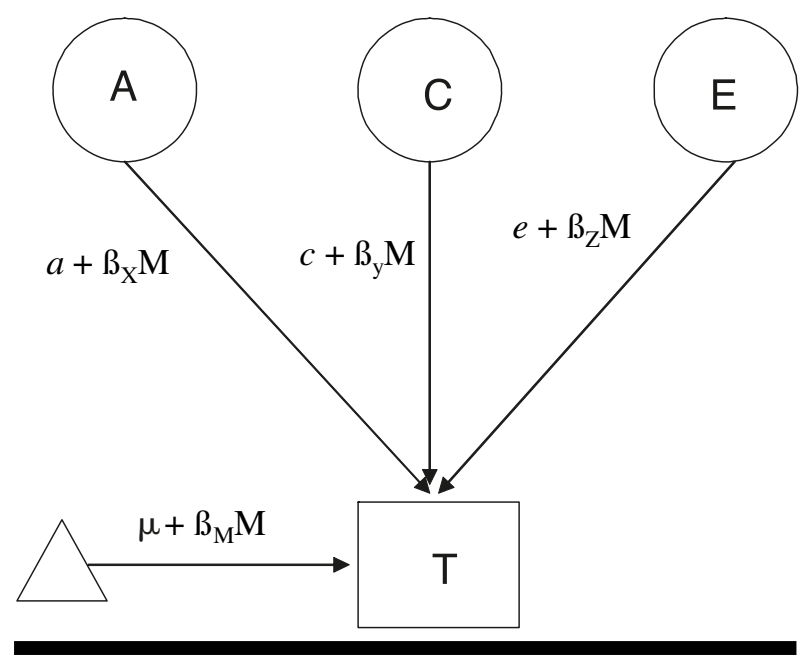

\section{Figure 1}

Gene-environment interaction model. The latent variable $A$ represented in a circle, indicates additive genetic influences on the trait $(T)$ of interest. $C$ represents common (shared) environmental influences on a trait, and latent $E$ represents unique environmental influences, which are uncorrelated between the twins. The triangle indicates the mean/thresholds for $T$ and is necessary when modeling raw data. The standard paths $a, c$, and $e$, indicating the magnitude of effect of each latent variable on the trait, each include a $\beta$ term, which indicates the significance of a potential environmental moderator $\mathrm{M}$ on each of these genetic and environmental influences. 
friends drank; $6 \%$ reported only one did; $28 \%$ reported that two to five friends did, and $48 \%$ reported that more than five did. The average friend alcohol use score was $3.1(S D=1.1)$.

\section{$\overline{\text { Data Analyses }}$}

\section{Twin Models Incorporating Moderation Effects}

In this paper we were interested in testing whether aspects of parenting and peer use have more complex effects on the development of substance use, interacting with and moderating the importance of genetic influences and other environmental risk factors. Figure 1 shows a classic twin model (for only one twin in the pair) that has been modified to include a moderation component. In the moderation model, the standard paths $a, c$, and $e$, indicating the magnitude of effects associated with additive genetic influences (A), common environmental influences $(\mathrm{C})$, and unique environmental influences (E), respectively, are allowed to vary as a function of a potential moderator variable. This is accomplished by including a $\beta$ term, which indicates the significance of a potential moderator $M$ on each of these genetic and environmental influences. In this way, the additive genetic path $(a)$ is extended to be a linear function of the moderator $M$, represented by the equation $a+\beta_{x} M$, where $\beta_{x}$ is an unknown parameter to be estimated from the data and represents the magnitude of the moderating effect. If $\beta_{x}$ is significantly different from zero, there is evidence for a moderating effect. A similar logic follows for the $\beta_{Y}$ and $\beta_{Z}$ terms, which represent the extent to which a specific moderator variable alters the importance of common and unique environmental influences, respectively. The pathway $\mu+\beta_{M} M$ models main effects of the moderator variable on the outcome. Also included in this pathway are any gene-environment correlation effects between the moderator variable and outcome. Thus, any covariance between the moderator and the outcome is removed by incorporating the moderator into the means model (Purcell, 2002; E. N. Turkheimer et al., 2003); accordingly, any interactions detected will be associated with the variance components unique to the outcome (i.e., genetic influences on smoking that are not shared with genetic influences on twins' reports of parenting). The value of the moderator $M$ is allowed to change from subject to subject, depending on the value of the moderator for that subject. $M$ can be obligatorily shared by the twins (i.e., a family-level variable) or vary between the twins (an individual level variable). Parental alcohol problems and smoking were obligatorily shared variables, as they were measured by parental report at the pair level. All other variables were made at the individual level, and each twin's individual-specific report was used in modeling.

Studying parenting within this framework raises some unique considerations, as studies have demonstrated that parental substance use is correlated with aspects of parenting practices, such as lower levels of parental support (Barnes \& Farrell, 1992; Holden et al., 1988), parental discipline (Tartar et al., 1993), and parental monitoring (Chassin et al., 1993). This raises concern that poor parenting practices may simply be a correlated third variable (with genetic influences on parent and child substance use), rather than an important risk factor in the development of the behavior. Although twin studies (where twins are the children reporting on their parenting, rather than twins being the parents themselves) are uninformative about the extent to which parental genotypes influence parenting (Purcell \& Koenen, 2005; Turkheimer et al., 2005), we did have information about the parents' substance use. Accordingly, if parenting characteristics per se were not an important factor, but rather, simply a proxy for parental substance use, we would expect that similar results might be obtained using parental substance use as a measured moderator. Accordingly, where the parenting variables of interest (monitoring, time spent with parents) showed evidence of significant moderating effects, we further explored these effects by fitting models using parental alcohol problems and parental smoking as the moderators on adolescent substance use to examine whether these variables might be involved in the interactions associated with parenting characteristics. Furthermore, we had information both on lifetime parental substance use and problems, which should reflect the predisposition of the parent (and to an extent, the child), and on current substance use by the parent(s), which reflects not only predisposition, but also the environment to which the child is currently exposed. Thus, although the traditional twin design is limited in the extent to which these variables can be disentangled, we compared the models using parenting characteristics and parenting substance use as moderators to aid in the interpretation of results.

\section{Interaction Between Moderators}

It is possible to further expand the basic moderator model to incorporate multiple moderators and test for interactions between them (Purcell, 2002). These models are quite complex and may have problems distinguishing between genetic and environmental moderation effects. Accordingly, as has been recommended (Purcell, 2002), we have restricted our use of these models to cases where (1) the moderating variables showed significant moderation effects when tested individually, and (2) we had specific hypotheses about the potential for interaction between moderators. Based on the parenting literature, we thought that it was important to test for interaction between parenting characteristics and parental substance use (for the cases where these variables showed individual moderation effects), based on evidence suggesting that the impact of parenting on adolescent substance use might vary according to the parents' own substance use. When two variables are incorporated into the multiple moderator geneenvironment interaction model, the genetic pathway 


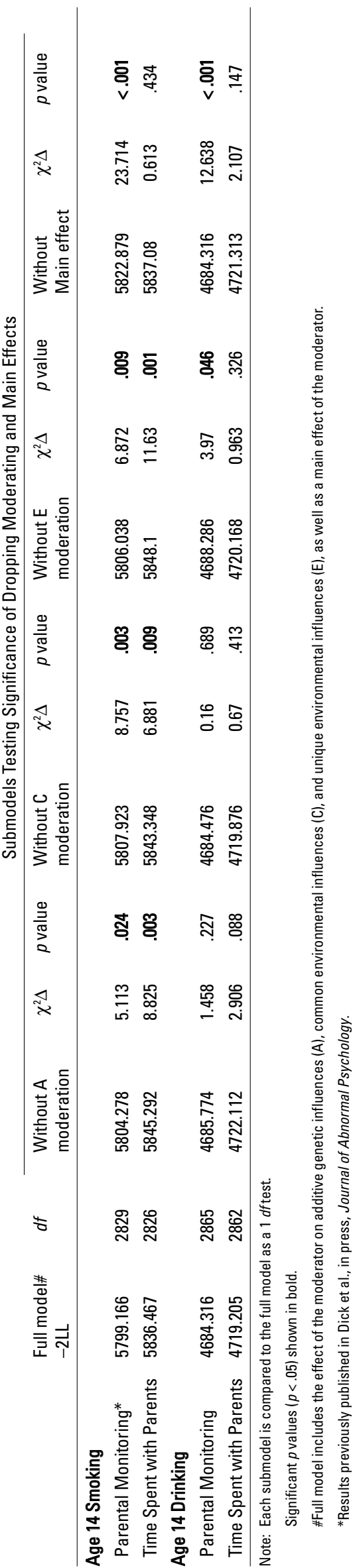

is now represented as $a+\beta_{M 1}(M 1)+\beta_{M 2}(M 2)+$ $\beta_{M 1} \times_{M 2}(M 1 \times M 2)$, where M1 was the parenting characteristic and M2 was the parental substance use variable in our models. Thus, the final $\beta$ coefficient $\left(\beta_{M 1 \times M 2}\right)$ allows us to test whether there is significant interaction between the two moderators. Moderating effects on the common and unique environmental pathways follow a parallel pattern.

All modeling was conducted using the raw ordinal data option in Mx (Neale et al., 1999). Mx is a structural equation modeling program developed specifically for the use of twin data. When the outcome is ordinal, the model involves the use of thresholds, rather than means. All moderating variables were standardized for analyses. The first application of the gene-environment interaction model using quasi-continuous environmental moderators was to the study of socioregional factors on alcohol use among young adults using Finnish twin data (Dick et al., 2001). These models have subsequently been detailed and expanded (Purcell, 2002). The significance of each of the parameters in the model can be tested by dropping a parameter and evaluating the change in -2 log likelihood between the initial model and the nested submodel. This difference is evaluated using a chi square distribution. A significant change in fit between the models $(p<.05)$ for the difference in degrees of freedom indicates that dropping the parameter caused a significant decrease in fit of the model, indicating that pathway significantly contributes to the outcome trait and should be retained in the model.

\section{Results}

Moderating Effects of Parenting Characteristics on Age 14 Substance Use

Both of the parenting variables, as measured at age 12 , were tested as a moderator of genetic and/or environmental effects on adolescent smoking and drinking at age 14. The fit statistics for these models are shown in Table 1. Parental monitoring had a significant main effect on both smoking and drinking; however, time spent in activities with parents did not have a main effect on either variable. Interestingly, adolescent smoking at age 14 appears to be more strongly influenced by the moderating effects of parenting than does adolescent drinking, as suggested by the fact that there were no significant moderating effects of parenting characteristics on adolescent drinking at this age, with the exception of the marginally significant moderation of $\mathrm{E}$ effects by parental monitoring (see discussion). Beyond that exception, there was no evidence that either of the parenting variables interacted with genetic and/or other environmental influences on adolescent drinking at age 14 .

In contrast, for adolescent smoking, significant evidence of interaction with both of the parenting variables was observed. Parallel to the results previously reported for parental monitoring and 


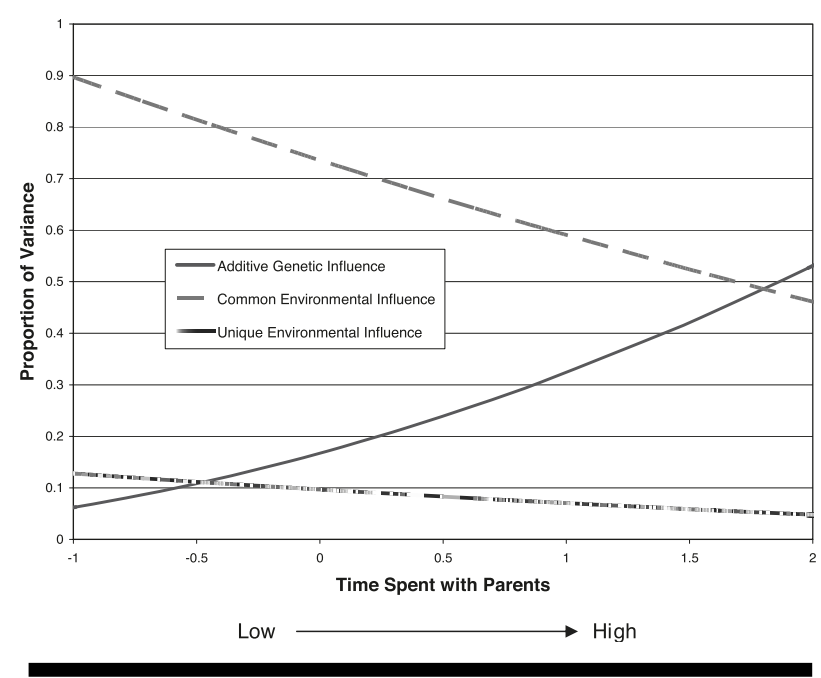

Figure 2

Changing variance in adolescent smoking at age 14 associated with additive genetic effects $(A)$, common environmental effects $(C)$, and unique environmental effects $(E)$ across increasing levels of time spent with parents.

adolescent smoking (Dick et al., in press), time spent in activities with parents also showed significant moderating effects. However, the direction of effect was opposite to that observed for parental monitoring. Figure 2 shows the changing proportions of variance attributed to genetic and environmental influences at different levels of time spent with parents. Genetic effects increased with increasing time spent with parents, and common and unique environmental effects decreased. Among adolescents who spent little time engaged in activities with their parents, $\mathrm{C}$ accounted for more than $85 \%$ of the variance in smoking, with little evidence of genetic influence. However, the proportion of variance attributed to genetic effects increased substantially with increasing time spent with parents, such that $\mathrm{A}$ accounted for just over $50 \%$ of the variance and $\mathrm{C}$ decreased to $\sim 45 \%$ of the variance at the extreme high end.

\section{Moderating Effects of Parental Substance Use}

We followed up the significant moderation effects associated with adolescent smoking by fitting models using the parental substance use variables as moderators of influences on adolescent smoking. These results are shown in Table 2. There were no significant moderating effects associated with lifetime parental alcohol problems or lifetime parental smoking (although both showed significant main effects, $p<.001)$. Interestingly, there were significant moderating effects associated with current parental substance use. With increasing levels of binge drinking by parents, and when the adolescents had a parent who currently regularly smoked, there was a significance increase in the magnitude of unique environmental influences on adolescent smoking. There was also a significant decrease (from $\sim 35 \%$ to $10 \%$ ) in the importance of genetic effects with more frequent binge drinking by a parent.

\section{Interaction between Parenting Characteristics and Parental Substance Use}

Finally, we tested for interaction between the two parenting characteristics that showed significant moderating effects, parental monitoring and time spent with parents, and the current parental smoking/drinking variables. There was no significant interaction on the moderation of $\mathrm{A}, \mathrm{C}$, or $\mathrm{E}$ components between the parenting characteristics and current parental substance use across any of the models (Table 3).

\section{Moderating Effects of Parenting Characteristics} on Age 17 Substance Use

To examine the extent to which the influence of parenting variables may change or remain consistent across adolescence, we fit parallel moderation models examining parental monitoring and time spent with parents, as measured at age 14 , as moderators of smoking and drinking frequencies, as measured at age 17. The moderation effects of genetic and common environmental influences observed between parental monitoring and smoking remained significant (dropping A moderation: $\chi^{2} \Delta=7.44,1 d f, p=.006$; dropping C moderation: $\chi^{2} \Delta=8.21,1 d f, p=.004$; dropping E moderation: $\left.\chi^{2} \Delta=0.12,1 d f, p=.73\right)$. Similarly, the moderation of $\mathrm{A}$ and $\mathrm{C}$ components between time spent with parents and smoking remained significant (dropping A moderation: $\chi^{2} \Delta=12.95,1 d f, p<.001$; dropping $\mathrm{C}$ moderation: $\chi^{2} \Delta=13.19,1 d f, p<.001$; dropping $\mathrm{E}$ moderation: $\left.\chi^{2} \Delta=0.15,1 d f, p=.70\right)$. Parallel to the results for age 14 alcohol use, there were no signifi-

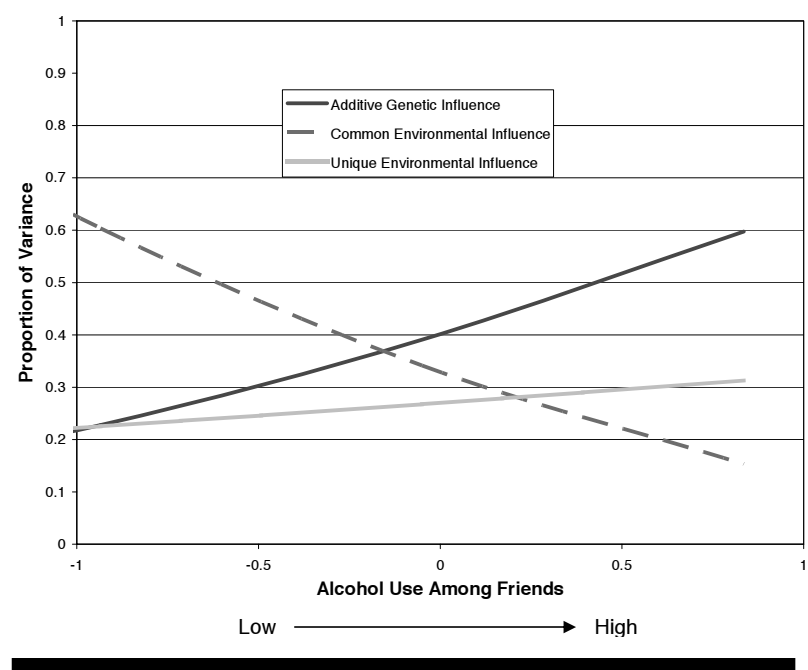

Figure 3

Changing variance in adolescent drinking at age 17 associated with additive genetic effects $(A)$, common environmental effects $(C)$, and unique environmental effects $(E)$ across increasing levels of friends' alcohol use. 
Table 2

Fit Statistics for Full Gene-Environment Interaction Model and Submodels Testing the Significance of Dropping each Parenting Substance Use Variable as a Moderating Variable on Influences on Adolescent Smoking

\begin{tabular}{|c|c|c|c|c|c|c|c|c|c|c|c|}
\hline & \multirow[b]{2}{*}{$\begin{array}{l}\text { Full model\# } \\
-2 \mathrm{LL}\end{array}$} & \multirow[b]{2}{*}{$d f$} & \multicolumn{9}{|c|}{ Submodels Testing Significance of Dropping Moderating Effects } \\
\hline & & & $\begin{array}{l}\text { Without } A \\
\text { moderation }\end{array}$ & $\chi^{2} \Delta$ & $p$ value & $\begin{array}{l}\text { Without C } \\
\text { moderation }\end{array}$ & $\chi^{2} \Delta$ & $p$ value & $\begin{array}{l}\text { Without } \mathrm{E} \\
\text { moderation }\end{array}$ & $\chi^{2} \Delta$ & $p$ value \\
\hline $\begin{array}{l}\text { Lifetime parental } \\
\text { alcohol problems }\end{array}$ & 5717.692 & 2755 & 5718.03 & 0.337 & .561 & 5718.41 & 0.718 & .397 & 5717.829 & 0.137 & .711 \\
\hline $\begin{array}{l}\text { Lifetime parental } \\
\text { regular smoking }\end{array}$ & 5599.015 & 2720 & 5599.887 & 0.872 & .35 & 5599.221 & 0.206 & .65 & 5599.334 & 0.319 & .572 \\
\hline $\begin{array}{l}\text { Current parental } \\
\text { binge drinking }\end{array}$ & 5882.023 & 2839 & 5886.786 & 4.763 & .029 & 5882.757 & 0.734 & .392 & 5886.916 & 4.893 & .027 \\
\hline $\begin{array}{l}\text { Current parental } \\
\text { regular smoking }\end{array}$ & 5328.225 & 2599 & 5329.265 & 1.04 & .308 & 5328.984 & 0.759 & .383 & 5333.214 & 4.989 & .026 \\
\hline
\end{tabular}

Note: Each submodel is compared to the full model as a $1 \mathrm{df}$ test.

\#Full model includes the effect of the moderator on additive genetic influences (A), common environmental influences (C), and unique environmental influences (E), as well as a main effect of the moderator.

cant moderation effects observed between parental monitoring or time spent with parents and age 17 frequency of alcohol use, with the exception that time spent with parents showed significant moderation of unique environmental effects (dropping E moderation: $\left.\chi^{2} \Delta=10.36,1 d f, p=.001\right)$.

\section{Moderating Effects of Friends' Alcohol Use on Age 17 Substance Use}

The lack of effects associated with parenting practices and adolescent alcohol use, despite the literature yielding strong evidence for significant environmental effects on adolescent alcohol use, led us to conduct initial analyses exploring whether other environmental influences might be more important on adolescent alcohol use. Accordingly, we tested for moderating effects associated with having a peer group who uses alcohol. Interestingly, we found significant moderation effects associated with peer alcohol use and adolescents' reports of their own frequency of alcohol use (full model: $-2 \mathrm{LL}=8253.96,2328 d f$; dropping A moderation: $\chi^{2} \Delta=12.58,1 d f, p<.001$; dropping C moderation: $\chi^{2} \Delta=17.12,1 d f, p<.001$; dropping E moderation: $\chi^{2} \Delta=6.31,1 d f, p=.01$ ). See Figure 3. There was also a significant main effect of friends' alcohol use $(p<.001)$. Although friends' alcohol use also had a significant main effect on smoking at age $17(p<.001)$, there were no significant moderation effects on smoking associated with peer alcohol use at age 17 .

\section{$\overline{\text { Discussion }}$}

Paralleling the movement toward identifying specific genes involved in behavior, there has been increasing interest in behavior genetics associated with identifying the specific environments that influence behavior, and how these environments interact with genetic predispositions. We believe this is a particularly important area in relation to adolescent substance use, where traditional twin models yield substantial evi- dence for the importance of environmental influences. Although there is a considerable literature on the role of parents and peers in adolescent substance use, most of this work has been conducted outside the context of genetically informative designs. Additionally, we believe that important effects associated with parenting and peers may be missed by studying these factors using a main effects framework. Accordingly, we have tested for potential moderation effects of parenting and peers on the relevance of genetic and environmental factors associated with adolescent substance use. Although these analyses are only a first step, and leave many remaining questions about how parental and peer influences are involved in adolescent substance use, we believe that they illustrate several interesting effects that warrant further exploration. They demonstrate that both parenting and peer characteristics can have strong and significant effects on adolescent substance use when tested within a more complex, interactive framework. However, we find that parenting has significant moderating effects only on adolescent smoking in our dataset, not on adolescent drinking, a finding which holds true for substance use measured both at ages 14 and 17. It does not appear that the moderation effects on adolescent smoking associated with parenting are merely a reflection of parental substance use problems. We do find some evidence that current parental substance use may also show moderating effects; however, there was no evidence of interaction between parenting behavior and parental substance use, as modeled here. Finally, despite the lack of evidence for moderating effects on adolescent alcohol use associated with parenting, we find significant evidence of moderation effects associated with friends' substance use on adolescent drinking at age 17.

Why might parenting characteristics show stronger moderation effects on adolescent smoking than on adolescent drinking? Interestingly, previous analyses of substance use at age 12 in this dataset, analyses that 
specifically distinguished between familial and extrafamilial sources of influence, found that familial household influences were more important on smoking at age 12, but that extrafamilial effects were more important for unsupervised drinking at this early age (Rose et al., 2003). The analyses of substance use at age 14 reported here, finding that parenting effects were more significantly associated with adolescent smoking, seem to further support our previous finding. It is also interesting that the effects associated with parenting on adolescent smoking remain significant from age 14 to age 17 , as this is consistent with our aforementioned finding from longitudinal analyses of smoking in FinnTwin16 that the common environmental influences on adolescent smoking are remarkably constant and unchanging across adolescence (Dick et al., 2006). In contrast, our previous longitudinal analyses of drinking frequency suggested that common environmental influences were more age-specific (Rose, Dick, Viken, \& Kaprio, 2001). Our preliminary analyses of peer alcohol use at age 17 suggest that, at least at this later stage in development, peers, rather than parents, may be more influential. This is in line with several previous studies that have found peer substance use to be one of the strongest risk factors for adolescent alcohol use (Marshal \& Chassin, 2000; Windle, 2000); as one example, in a study of over 570 middle adolescents, peer and sibling substance use were more strongly related to adolescent substance use than parental alcohol use was (Windle, 2000).

Another interesting finding to emerge from these analyses is that different dimensions of parenting appear to have different effects on outcome. In relation to parental monitoring, we found that genetic influences on adolescent smoking decreased and common environmental influences increased at higher levels of parental monitoring. These analyses suggest that when adolescents receive little parental monitor- ing, it creates an environment that allows for greater opportunity to express genetic predispositions. The moderating effects of peer alcohol use on adolescent drinking operated in a similar fashion: among adolescents with a larger number of peers who used alcohol, there was greater expression of genetic predispositions. These findings may reflect a situation whereby environments characterized by low parental monitoring or high peer substance use create opportunity for adolescents to express genetic predispositions. These results are in line with previous findings from the Finnish twin studies, which indicated that in neighborhoods where there is less stability, presumably engendering less community monitoring, there was greater evidence of genetic influence (Dick et al., 2001). Conversely, in more supervised and restricted environments, there was less opportunity to express genetic predispositions and greater influence of environmental effects (Dick et al., 2001; Rose, Dick, Viken, \& Kaprio, 2001).

In contrast, significant moderating effects for time spent engaged in activities with parents were also observed, but this parenting characteristic operated in a different manner. As adolescents reported spending more time with their parents there was an increase in genetic variance. These findings suggest that spending more time with biologically related relatives may engender the expression of genetic predispositions. An implication of this finding is that, for some children, spending time with parents may be beneficial, but for other children, it may not, depending on the behavior and predispositions of the parents. This supports a previous report that children who spent more time with their parents were less likely to become involved in alcohol-related behaviors only when parental alcohol use was not taken into account; children who spent more time with alcohol-using parents were actually more likely to use alcohol (Yu, 2003). This also may explain the lack of a main effect associated

\section{Table 3}

Fit Statistics for Gene-Environment Interaction Models Incorporating Interaction Between Two Moderating Variables

\begin{tabular}{|c|c|c|c|c|c|c|c|c|c|c|c|}
\hline & \multirow[b]{2}{*}{$\begin{array}{l}\text { Full model\# } \\
-2 \mathrm{LL}\end{array}$} & \multirow[b]{2}{*}{ f df } & \multicolumn{9}{|c|}{ Submodels Testing Significance of Dropping Moderating Interaction Effects } \\
\hline & & & $\begin{array}{l}\text { Without A } \\
\text { interaction }\end{array}$ & $\chi^{2} \Delta$ & $p$ value & $\begin{array}{l}\text { Without C } \\
\text { interaction }\end{array}$ & $\chi^{2} \Delta$ & $p$ value & $\begin{array}{l}\text { Without } \mathrm{E} \\
\text { interaction }\end{array}$ & $\chi^{2} \Delta$ & $p$ value \\
\hline $\begin{array}{l}\text { Monitoring } \\
- \text { Binge drinking }\end{array}$ & 5720.385 & 2800 & 5721.072 & 0.687 & .407 & 5720.387 & 0.002 & .96 & 5720.385 & $<0.001$ & ns \\
\hline $\begin{array}{l}\text { Monitoring } \\
\text { - Smoking }\end{array}$ & 5195.975 & 2569 & 5196.045 & 0.07 & .791 & 5195.98 & 0.005 & .941 & 5196.651 & 0.676 & .411 \\
\hline $\begin{array}{l}\text { Time with parents } \\
\text { - Binge drinking }\end{array}$ & 5751.158 & 2797 & 5751.613 & 0.455 & .5 & 5751.222 & 0.065 & .799 & 5751.186 & 0.029 & .865 \\
\hline $\begin{array}{l}\text { Time with parents } \\
\text { - Smoking }\end{array}$ & 5220.932 & 2564 & 5222.414 & 1.482 & .223 & 5223.51 & 2.578 & .108 & 5221.01 & 0.078 & .78 \\
\hline
\end{tabular}

Note: Fit Statistics are shown for the full model and each submodel testing the significance of dropping the moderation interaction effects $\left(\beta_{\mathrm{M} 1} \times_{\mathrm{M} 2}\right)$ for each component of variance. Each submodel is compared to the full model as a $1 \mathrm{df}$ test.

\#Full model includes an effect of each moderator variable on additive genetic influences (A), common environmental influences (C), and unique environmental influences (E), interaction between the two moderators on $A, C$, and $E$, as well as main effects of the moderators. 
with this variable in our dataset. We did not find any evidence of interaction between time spent with parents and parental substance use here; however, these moderation interaction models are generally underpowered and remain exploratory. Our finding of increased genetic effects with increased time spent with parents is consistent, as well, with a previous study by Jaffee and colleagues (2003), in which they concluded that being raised by two biological parents was not always beneficial; rather, it depended on the characteristics of the parents. Children who resided with antisocial fathers were more likely to manifest conduct problems, as they received a 'double whammy' of genetic and environmental risk (Jaffee et al., 2003). The results from our data suggest that parents who do not spend time engaged in activities with their children leave them to their own devices, resulting in greater influence of environmental effects (presumably extrafamilial) under these circumstances. Finally, we note that time spent with parents and parental monitoring show a low correlation in our dataset $(<.15)$; accordingly, it is not altogether surprising that these aspects of parenting operate in different manners. We are currently conducting follow-up analyses to explore potential interaction between these two dimensions of parenting.

In all cases where there was significant moderation of unique environmental effects, the direction of effect was the same: unique environmental influences decreased with increasing levels of parental monitoring, time spent with parents, and current parental substance use. These findings suggest that with increasing levels of parental involvement and decreasing levels of parental substance use, adolescents become less susceptible to the 'slings and arrows of outrageous fortune'; random effects are less likely to impact their patterns of substance use. One may also note that the $p$ value attached to the moderation of $\mathrm{E}$ effects is often much more significant than that associated with $\mathrm{A}$ or $\mathrm{C}$ moderation effects, despite the changing level of proportion of variance being smaller. This is due to the low power of the twin model to discriminate between $\mathrm{A}$ and $\mathrm{C}$ effects, as they both have the effect of making siblings more similar to one another (Neale et al., 1994). Because E effects operate in the opposite direction, and are the only source of variance that makes both types of twins different from one another, there is greater power to detect these effects. We conducted power analyses prior to initiating analyses in the FT data, as our analyses represent one of the first applications, to our knowledge, of moderation models with ordinal data. Our analyses suggested that the FT12 sample had adequate power $(>80 \%)$ to detect significant $\mathrm{A}$ interaction effects, even when the outcome variable was highly skewed. Thus, although the power to detect $\mathrm{A}$ and $\mathrm{C}$ interaction effects is reduced compared to E effects, adequate power remained in this sample to detect interaction effects of moderate size $(\beta=.4)$.

There are several limitations of this study. The moderators studied here, parenting and peer characteristics, are themselves likely to be genetically influenced. In relation to the parenting variables, with data only on twin children and their parents, we are unable to study the extent to which (possibly overlapping) genetic predispositions in the parents contributed to their parenting practices and substance use, and were transmitted to their children. We have attempted to investigate this issue by comparing models fit to both parenting characteristics and parental substance use, and we believe that it is informative that only parenting characteristics and current parental substance use (not lifetime, which may be more reflective of underlying predispositions than environmental exposure) moderate influences on adolescents' smoking. Although this study is uninformative as to the extent to which genetic factors influenced the parents' parenting practices, we believe the finding that parenting - to the extent that it reflects both genetic predispositions and environmental context - dramatically influences the degree to which genetic predispositions are expressed in children and the extent to which random environmental factors impact adolescents' smoking practices, is an important result. Complementary study designs must also be used to better understand the effects of parenting and how parenting characteristics act and interact with children's genetic predispositions. One study design that is particularly informative for elucidating causal relations between parent characteristics and child outcomes is the children of twins design (D'Onofrio et al., 2003). In relation to the moderation effects associated with friends' alcohol use, we know that friends' alcohol use is also under significant genetic influence in our data (Pagan et al., June 2006), presumably reflecting, in part, selective processes involved in friendship formation. As detailed in the methods, any gene-environment correlation between the moderator and outcome is modeled in the main effects term; accordingly, the moderation effects detected reflect moderation of the variance components unique to alcohol drinking frequency.

Second, we were unable to test whether parenting effects on substance use differed between adolescent boys and girls. The complexity of these gene-environment interaction models requires large sample sizes to detect significant interaction, particularly when both $\mathrm{A}$ and $\mathrm{C}$ effects are involved in the outcome, as is the case with adolescent substance use, and when the outcome measure is ordinal. To achieve sufficient power to detect these effects, we collapsed across sex. However, for the significant gene-environment interaction effects that were detected, for parental monitoring and time spent with parents on adolescent smoking, we did fit the models separately to male and female data. Although this did not allow us 
to formally test for sex differences, it did allow us to examine the pattern of results separately by gender for suggestions of possible sex differences. In general, the results obtained from these sex-specific analyses looked very similar for males and females to the overall results obtained from the full sample.

In conclusion, we have studied the effects of two dimensions of parenting (parental monitoring and time spent in activities with parents), on patterns of substance use among adolescents at age 14 and age 17 , using a genetically informative design. We find strong moderating effects associated with parenting characteristics on adolescent smoking, but not on adolescent drinking. These results are consistent from age 14 to age 17 . Conversely, we find evidence of moderating effects of peer substance use on adolescent drinking at age 17. These analyses illustrate the importance of incorporating measured aspects of the environment into genetically informative twin models to begin to understand how specific environments are related to various outcomes. Furthermore, they illustrate the importance of taking a developmental perspective in studies of this nature to understand how specific influences may vary across different ages, and across different phenotypes.

\section{Acknowledgments}

Preparation of this manuscript was supported by AA015416 to DMD. FinnTwin12 has been supported by the National Institute of Alcoholism and Alcohol Abuse (grants AA-12502, AA-00145, and AA-09203 to RJR), and by the Academy of Finland (to JK), the Finnish Centre of Excellence Programme (to LP and JK) and grants from the Yrjö Jahnsson Foundation (to JK).

The authors acknowledge funding support from the National Institute on Aging. The views expressed are those of the authors of this paper, and not the National Institute on Aging, the National Institutes of Health or the Department of Health and Human Services.

\section{References}

Barnes, G. M., \& Farrell, M. P. (1992). Parental support and control as predictors of adolescent drinking, delinquency, and related problem behaviors. Journal of Marriage and the Family, 54, 763-776.

Barnes, G. M., Reifman, A. S., Farrell, M. P., \& Dintcheff, B. A. (2000). The effects of parenting on the development of adolescent alcohol misuse: A sixwave latent growth model. Journal of Marriage and the Family, 62, 175-186.

Boomsma, D. I., van Beijsterveldt, C. E., \& Hudziak, J. J. (2005). Genetic and environmental influences on anxious/depression during childhood: A study from the Netherlands Twin Register. Genes, Brain, and Behavior, 4, 466-481.

Borawski, E., Levers-Landis, C., Lovegreen, L., \& Trapl, E. (2003). Parental monitoring, negotiated unsupervised time, and parental trust: The role of perceived parenting practices in adolescent health risk behaviors. Journal of Adolescent Health, 33, 60-70.

Bouchard, T. J., Jr., \& McGue, M. (2003). Genetic and environmental influences on human psychological differences. Journal of Neurobiology, 54, 4-45.

Chassin, L., Pillow, D., Curran, P., Molina, B., \& Barrera, M. (1993). Relation of parental alcoholism to early adolescent substance use: A test of three mediating mechanisms. Journal of Abnormal Psychology, 102, 3-19.

Dick, D. M., Barman, S., \& Pitkanen, T. (2006). Genetic and environmental influences on the initiation and continuation of smoking and drinking. In L. Pulkkinen, J. Kaprio \& R. J. Rose (Eds.), Socioemotional development and health from adolescence to adulthood (pp. 126-145). New York: Cambridge University Press.

Dick, D. M., Purcell, S., Viken, R. J., Kaprio, J., Pulkkinen, L., \& Rose, R. J. (in press). Parental monitoring moderates the importance of genetic and environmental influences on adolescent smoking. Journal of Abnormal Psychology.

Dick, D. M., \& Rose, R. J. (2002). Behavior Genetics: What's new? What's next? Current Directions in Psychological Science, 11, 70-74.

Dick, D. M., Rose, R. J., Viken, R. J., Kaprio, J., \& Koskenvuo, M. (2001). Exploring gene-environment interactions: Socioregional moderation of alcohol use. Journal of Abnormal Psychology, 110, 625-632.

D’Onofrio, B. M., Turkheimer, E. N., Eaves, L. J., Corey, L. A., Berg, K., Solaas, M. H., et al. (2003). The role of the children of twins design in elucidating causal relations between parent characteristics and child outcomes. Journal of Child Psychology and Psychiatry, 44, 1130-1144.

Goldsmith, H. H. (1991). A zygosity questionnaire for young twins: A research note. Behavior Genetics, 21, 257-269.

Hjelmborg, J. V., Iachine, I., Skytthe, A., Vaupel, J. W., McGue, M., Koskenvuo, M., Kaprio, J., Pedersen, N. L., \& Christensen, K. (2006). Genetic influences on human lifespan and longevity. Human Genetics, 119, 312-321.

Holden, M. G., Brown, S. A., \& Mott, M. A. (1988). Social support network of adolescents: Relation to family alcohol abuse. American Journal of Drug and Alcohol Abuse, 14, 487-498.

Jaffee, S. R., Moffitt, T. E., Caspi, A., \& Taylor, A. (2003). Life with (or without) father: The benefits of living with two biological parents depend on the father's antisocial behavior. Child Development, 74, 109-126.

Kaprio, J., Pulkkinen, L., \& Rose, R. J. (2002). Genetic and environmental factors in health-related behaviors: Studies on Finnish twins and twin families. Twin Research, 5, 358-365. 
Kaprio, J., Rimpela, A., Winter, T., Viken, R. J., Rimpela, M., \& Rose, R. J. (1995). Common genetic influences on BMI and age at menarche. Human Biology, 67, 739-753.

Kerr, M., \& Stattin, H. (2000). What parents know, how they know it, and several forms of adolescent adjustment: Further support for a reinterpretation of monitoring. Developmental Psychology, 36, 366-380.

Klump, K. L., McGue, M., \& Iacono, W. G. (2003). Differential heritability of eating attitudes and behaviors in prepubertal versus pubertal twins. International Journal of Eating Disorders, 33, 287-292.

Marshal, M. P., \& Chassin, L. (2000). Peer influence on adolescent alcohol use: The moderating role of parental support and discipline. Applied Developmental Science, 4, 80-88.

Narusk, A., \& Pulkkinen, L. (1994). Parental relationships and adolescents' conceptions of their interaction with significant others. European Journal of Psychology of Education, 9, 203-213.

Neale, M. C., Boker, S. M., Xie, G., \& Maes, H. H. (1999). Mx: Statistical Modeling (Version 5th Edition). Box 126 MCV, Richmond, VA 23298: Department of Psychiatry.

Neale, M. C., Eaves, L. J., \& Kendler, K. S. (1994). The power of the classical twin study to resolve variation in threshold traits. Behavior Genetics, 24, 239-258.

Pagan, J. L., Holliday, C., Rose, R. J., Viken, R. J., Pulkkinen, L., Kaprio, J., et al. (June 2006). The role of peers in substance use initiation in early adolescence. Paper presented at the Annual Meeting of the Behavior Genetics Association, Storrs, CT.

Pulkkinen, L., Kaprio, J., \& Rose, R. J. (1999). Peers, teachers, and parents as raters of twins' behavioural and emotional problems of twins and their adjustment: The Multidimensional Peer Nomination Inventory. Twin Research, 2, 274-285.

Purcell, S. (2002). Variance components models for gene-environment interaction in twin analysis. Twin Research, 5, 554-571.

Purcell, S., \& Koenen, K. (2005). Environmental mediation and the twin design. Behavior Genetics, 35, 491-498.

Rai, A., Stanton, B., Wu, Y., Xiaoming, L., Galbraith, J., Cottrell, L., Pack, R., Harris, C., D’Alessandri, D., \& Burns, J. (2003). Relative influences of perceived parental monitoring and perceived peer involvement on adolescent risk behaviors: An analysis of six crosssectional data sets. Journal of Adolescent Health, 33, 108-118.
Rimpela, M., Rimpela, A., Rahkonen, O., \& Teperi, J. (1988). The evolution of the juvenile health habit study 1977-1978. In R. Anderson, J. K. Davies, I. Kickbusch, D. V. McQueen \& J. Turner (Eds.), Health behaviour research and bealth promotion (pp. 154-171). New York: Oxford University Press.

Rose, R. J., Dick, D. M., Viken, R. J., \& Kaprio, J. (2001). Gene-environment interaction in patterns of adolescent drinking: Regional residency moderates longitudinal influences on alcohol use. Alcoholism: Clinical and Experimental Research, 25, 637-643.

Rose, R. J., Dick, D. M., Viken, R. J., Pulkkinen, L., \& Kaprio, J. (2001). Drinking or abstaining at age 14: A genetic epidemiological study. Alcoholism: Clinical and Experimental Research, 25, 1594-1604.

Rose, R. J., Kaprio, J., Winter, T., Koskenvuo, M., \& Viken, R. J. (1999). Familial and socio-regional environmental effects on abstinence from alcohol at age 16. Journal of Studies on Alcohol, Supplement No. $13,63-74$.

Rose, R. J., Viken, R. J., Dick, D. M., Bates, J., Pulkkinen, L., \& Kaprio, J. (2003). It does take a village: Nonfamilial environments and children's behavior. Psychological Science, 14, 273-277.

Seppa, K., Sillanaukee, P., \& Koivula, T. (1990). The efficiency of a questionnaire in detecting heavy drinkers. British Journal of Addiction, 85, 1639-1645.

Stattin, H., \& Kerr, M. (2000). Parental monitoring: A reinterpretation. Child Development, 71, 1072-1085.

Steinberg, L., Fletcher, A., \& Darling, N. (1994). Parental monitoring and peer influences on adolescent substance use. Pediatrics, 93, 1060-1064.

Tartar, R. E., Blackson, T., Martin, C., Loeber, R., \& Moss, H. B. (1993). Characteristics and correlates of child discipline practices in substance abuse and normal families. The American Journal on Addictions, $2,18-25$.

Turkheimer, E., D’Onofrio, B. M., Maes, H. H., \& Eaves, L. J. (2005). Analysis and interpretation of twin studies including measures of the shared environment. Child Development, 76, 1217-1233.

Turkheimer, E. N., Haley, A., Waldron, M., D’Onofrio, B. M., \& Gottesman, I. I. (2003). Socioeconomic status modifies heritability of IQ in young children. Psychological Science, 14, 623-628.

Windle, M. (2000). Parental, sibling, and peer influences on adolescent substance use and alcohol problems. Applied Developmental Science, 4, 98-110.

Yu, J. (2003). The association between parental alcoholrelated behaviors and children's drinking. Drug and Alcohol Dependence, 69, 253-262. 